\title{
ARBEITSSUCHT UND BURNOUT- SYNDROM - AKTUELLE RISIKOFAKTOREN DES MANAGERS DER GEGENWART
}

\author{
Jan Molek, Ing. CSc. \\ Jihočeská univerzita v Českych Budějovicích, Zdravotně sociálni fakulta \\ Address: $\quad$ J.Boreckeho 27, 37011 České Budějovice, Tel..389037563 \\ E-Mail: $\quad$ molek@zsf.jcu.cz
}




\title{
ARBEITSSUCHT UND BURNOUT- SYNDROM - AKTUELLE RISIKOFAKTOREN DES MANAGERS DER GEGENWART
}

\begin{abstract}
Summary
Workoholism is often considered to be positive and beneficial and workoholics are supposed to be very efficient people, who love their own work and are not lazy. In fact, workoholism is not connected with enthusiasm at all but is a dependency like any other. People dealing day-to-day with other people (teachers, doctors etc.) or those having significant responsibility (managers) suffer from the consequences of excessive work-load the most. As a result of exhaustion, people feel dissatisfaction and do not enjoy their work at the beginning. This is followed by minor health problems along with decreasing work efficiency. Finally, people's self-confidence and energy is reduced significantlty.

Burnout threatens managers the most. Psychologists claim the major reason is managers' reluctancy or inability to delegate tasks to their subordinate colleagues. Instead, they prefer to carry out as many tasks as possible on their own to ensure the required quality. Burnout is the result of total exhaustion and a simple reduction in activity does not solve the problem.
\end{abstract}

Key words: workoholism, dependency, retrieval, burnout

\section{EINLEITUNG}

Unter dem Begriff krankhafte Abhängigkeit stellen sich die Menschen in der Regel „klassische" Drogen vor, angefangen beim Alkohol bis hin zum Heroin. Psychische und physische Probleme werden jedoch nicht nur durch Suchtmittel verursacht, sondern auch durch Suchtverhalten, dem die heutige Zeit leider außerordentlich förderlich ist. Und den Prognosen der Soziologen nach zu urteilen, wird dies wahrscheinlich auch in nächster Zukunft nicht viel besser werden. Die Folgen dürfen keinesfalls auf die leichte Schulter genommen werden, denn sie sind meist sehr ernst und betreffen oft nicht nur die abhängige Person selbst, sondern auch ihr nahe stehende Personen.

$\mathrm{Zu}$ den heute meist verbreiteten Süchten gehört Arbeitssucht, d.h. die krankhafte Abhängigkeit von der Arbeit. Eine der schwerwiegendsten potentiellen Folgen dieser Abhängigkeit (Krankheit) ist das Syndrom der Ausbrennung (Burnout-Syndrom).

Diese Vorlesung dient keinesfalls als eine Abhandlung über Arbeitssucht und BurnoutSyndrom aus medizinischer Sicht, sondern zur Betrachtungsweise dieses Problems unter dem Blickwinkel der Praxis in den Firmen.

\section{ARBEITSSUCHT SCHADET DEN MENSCHEN UND DER FIRMA}

Die krankhafte Abhängigkeit von der Arbeit (Arbeitssucht) betrifft immer mehr Menschen, insbesondere aus den Reihen der Führungskräfte. Manch einer hält ein übertriebenes Arbeitsengagement für eine Tugend, in Wirklichkeit handelt es sich aber um eine Krankheit mit unerträglichen Auswirkungen auf das Individuum und die Organisation. 


\subsection{Arbeitssucht ist eine tückische Krankheit der heutigen Zeit}

Ein von der Arbeit abhängiger Mensch überschreitet ständig seine körperlichen und geistigen Grenzen, oftmals ist er nicht imstande, auszuruhen, und wenn er ausruht, fühlt er sich schuldig. Er will alles (oder so viel wie möglich) selbst erledigen und bei Teamarbeiten hat er meist Probleme. Er stellt sich Termine, die er nur deshalb versucht krampfhaft zu erfüllen, weil er sich dann beim nächsten Mal noch kürzere Termine setzen kann. Er ist immer in Eile, auch wenn es dafür keine Gründe gibt. Er ist unrealistisch und verspricht oft mehr, als er schaffen kann. Solche Menschen nutzen die Arbeit häufig dazu, um unangenehmen seelischen Empfindungen und unangenehmen Tatsachen der heutigen Welt zu entfliehen. Sie gestatten weder sich noch anderen, müde zu sein, und gebrauchen oft gefährliche Methoden, um die übermäßige Belastung in Griff zu bekommen (Alkohol, Zigaretten und andere Suchtmittel). In der Regel vernachlässigen sie nicht nur ihre Gesundheit (was sich darin äußert, dass sie bei gesundheitlichen Problemen meistens nicht einmal einen Arzt aufsuchen),sondern sie vernachlässigen auch die zwischenmenschlichen Beziehungen (sie überfordern sich vor allem nur dann, wenn ihnen das irgendeinen Vorteil in der Arbeit verschafft). Sie sind nur auf ihre Arbeitsziele fokussiert und das, was andere erleben, verstehen sie nicht oder es interessiert sie nicht. Sie pflegen ungeduldig und gereizt zu sein und wegen kleinster Fehler sind sie übertrieben wütend auf sich selbst. Sie haben entweder einen Hang zu Selbstmitleid, oder ignorieren ihre Gefühle einfach. Häufig gebärden sie sich unwahrscheinlich ernst und leiden unter dem Eindruck, dass sie von anderen nicht geschätzt werden. Nicht selten führen sie einen chaotischen und unorganisierten Lebensstil und sind meist einsam.

Während sich niemand stolz zu einer Abhängigkeit von Alkohol oder Drogen bekennt, sind auch diejenigen stolz auf ihre Arbeitssucht, die noch weit von ihr entfernt sind. Weshalb wollen so viele Menschen Workaholic sein und sind dabei über jeden Zweifel an ihrer Arbeitsauslastung erhaben? Es geht doch um eine gefährliche Sucht, die bis zum Zerfall der Persönlichkeit und zum totalen Zusammenbruch führen kann. Aus einem normalen Menschen wird ein Egoist, der Tendenzen hat, andere zu beherrschen und sie zu manipulieren. Ein Mensch, der alles kontrolliert und niemandem traut. Außerdem verliert er den Sinn für Werte und auch den Humor, der das Leben doch verlängern soll. Er verliert die Fähigkeit, zu kommunizieren und bleibt allein. Man kann sagen, er wird zu einem asozialen Mitglied der Gesellschaft. 


\subsection{Wo endet Arbeitsengagement und beginnt Arbeitssucht}

Arbeitssucht fügt sich der Mensch selbst zu und zwar durch falsches Herantreten an die Arbeit. Wo ist aber diese imaginäre Grenze, an der das Arbeitsengagement endet und Arbeitssucht beginnt? Zahlreiche Fachleute sind sich darin einig, dass aus Arbeitsengagement Arbeitssucht wird, wenn der Mensch:

- immer mehr Zeit in der Arbeit verbringt aber ständig das Gefühl hat, dass die Arbeitsaufgaben einfach nicht weniger werden.

- nicht aufhört, an seine Arbeit zu denken und sich in jeder Form bemüht, alle (oft auch nebensächliche) Aufgaben zu erfüllen.

- die meiste Zeit mit unwichtigen Tätigkeiten verbringt und sich ihm das strategische Ziel $\mathrm{zu}$ entfernen scheint.

Wie erkennt der Mensch nun aber, dass er wirklich arbeitssüchtig (also ein Workaholic) ist? Ausgangspunkt ist die Beantwortung folgender Fragen:

- Sind sie unruhig und unkonzentriert, wenn sie gerade nicht arbeiten?

- Sehen sie es ausgesprochen ungern, wenn sie jemand bei der Arbeit stört?

- Stört die Arbeit ihr Familien- und gesellschaftliches Leben?

- Haben sie alle Ihre Vorlieben und edelsinnigen Aktivitäten durch die Arbeit ersetzt?

- Stören arbeitsfreie Wochenenden und Feiertage ihr psychisches Leben?

- Sind sie nicht in der Lage, das festgelegte Maß ihrer Tätigkeit einzuhalten?

Eine positive Beantwortung der meisten Fragen sollte für sie ein Impuls sein, über sich und ihre eigenen Angewohnheiten nachzudenken.

\subsection{Wie wird man Workaholic}

$\mathrm{Zu}$ einem Workaholic kann ein Mensch auch dann werden, wenn er das Ziel seiner Anstrengungen (Bemühungen) zwar definieren, jedoch nicht geradewegs zu ihm gelangen kann. Hat er keine Autorität an seiner Seite, die ihn lenken und positiv beeinflussen kann, begeht er auf dem Weg zu seinem Ziel viele Versehen und Fehler, die sukzessive untrennbarer Bestandteil seiner Arbeit werden (häufig geht er uneffektiv vor und schlägt Wege ein, die in Sackgassen führen; die Erledigung nebensächlicher Arbeiten verdrängt schrittweise die strategischen Aufgaben (infolge dessen sich das Ziel zu entfernen beginnt). Früher oder später fängt er an, in seiner eigenen Arbeit herumzuirren. Das Verlangen, das erträumte Ziel zu erreichen, ist zwar sehr stark und die Nervosität ruft auf Grund der 
Tatsache, dass das Ziel immer mehr in die Ferne rückt, noch größere Anstrengungen hervor, doch das Ergebnis besteht meist nur darin, dass sich das Ziel etwas langsamer entfernt.

$\mathrm{Zu}$ einem Workaholic wird vor allem ein arbeitsamer Mensch. Und deshalb muss er sich, bevor er sich ansteckt, ein Arbeitssystem schaffen und die Arbeitsergebnisse dann permanent verfolgen und auswerten.

\subsection{Mythen über Workaholics}

Ähnlich wie viele andere krankhafte Süchte ist auch der Arbeitssucht von Mythen umgeben, die es nicht gerade leichter machen, das wahre Wesen der Krankheit und die Heilungsmöglichkeiten zu erkennen. Einige der bekanntesten Mythen über Workaholics sind zum Beispiel:

\section{- Sie sind erfolgreicher.}

Irrtum! Länger und härter als jeder andere zu arbeiten, ist nicht der richtige Weg zum Erfolg. Die Guten und Erfolgreichen arbeiten einfach besser und schneller.

\section{- Die Chefs mögen sie.}

Bloße Illusion! Gute Chefs haben in Wirklichkeit ihre Befürchtungen, was einen Workaholic und dessen Arbeit im Team anbelangt. Workaholics sind ein Problem.

\section{- Sie sind vom Streben nach Erfolg motiviert.}

Falsch! Workaholics sind gewöhnlich durch Angst motiviert - durch Angst vor Konkurrenz, Angst vor Versagen in der Arbeit oder durch Angst vor der realen Welt außerhalb ihres Arbeitsplatzes.

\section{- Sie haben die Sachen unter Kontrolle.}

Das Gegenteil ist richtig! Wenn Workaholics die Sachen unter Kontrolle hätten, müssten sie nicht so hart - härter als die anderen - arbeiten.

\section{- Sie machen weniger Fehler.}

Unsinn! Weil sie länger und intensiver als die andere arbeiten, sind sie müder und haben somit mehr Zeit nicht nur mehr Fehler zu machen, sondern diese auch immer wieder von neuem zu machen.

\section{- Sie können nichts dafür.}

Das ist eine Ausrede! Arbeitssucht ist eine Sucht, die man beherrschen und heilen kann (wenn es ein Workaholic „,wirklich" will). 


\subsection{Wie man der Arbeitssucht vorbeugen kann}

Ähnlich wie andere Süchte entsteht der Arbeitssucht allmählich und mehr oder weniger unbewusst. Der Mensch arbeitet gern, die Arbeit bereitet ihm Freude, er schätzt seine Arbeit und oftmals wird ihm gar nicht bewusst, dass er von ihr abhängig ist. Damit erhebt sich die legitime Frage: Wie lässt sich der Arbeitssucht vorbeugen?

Das Mittel gegen Arbeitssucht ist ein gut funktionierendes Arbeitssystem. Das bedeutet in der Praxis:

- Festlegung wirksamer Regeln und Applikation eines effektiven Arbeitssystems,

- Planung und Auswertung der erreichten Ergebnisse,

- Selbstrealisierung, geistige Hygiene und Selbstkontrolle.

Arbeit ist für das Leben eines gesunden und kreativen Individuums notwendig, da sie das Leben erfüllt und bereichert. Der Arbeit darf aber nicht absolute Bedeutung und Zeit beigemessen werden. Für die Arbeit, aber auch für die Familie, Freunde, Hobbys, Weiterbildung, körperliche Aktivitäten u. ä. muss die entsprechende Zeit eingeplant werden. Diese Tätigkeiten sollten nach Meinung zahlreicher Experten maximal zwei Drittel des Tages ausfüllen. Das restliche Drittel muss für Ruhe, Erholung, Zeitverluste, Reserven und für unvorhersehbare Umstände vorbehalten werden.

Unbedingte Voraussetzung für hohe Leistungskraft eines Menschen (insbesondere eines Menschen, der in die Risikogruppe des Personals fällt) ist eine gute psychische und körperliche Kondition (nur im Zustand körperlichen und geistigen Wohlbefindens kann man nicht nur seine eigene Zeit, sondern auch die Zeit seiner Mitarbeiter effizient managen).

Eine effektive Methode, wie geistige Ausgeglichenheit von Führungskräften erzielt werden kann, ist die Strategie $\boldsymbol{A I D} \boldsymbol{A}$, die eine Anleitung dafür ist, wie Probleme und Konflikte in der Firma ohne Risiko, die eigene Gesundheit zu gefährden, erfolgreich gelöst werden können. Das Prinzip dieser Strategie erklären Teplickä und Kamenikovä (Modernes Management, 2004) wie folgt: 


\begin{tabular}{|cl|l|}
\hline A & Aufmerksamkeit & aktuellen und vorrangigen Aufgaben widmen. \\
\hline I & Interesse & $\begin{array}{l}\text { der Sphäre widmen, die den Menschen interessiert und } \\
\text { ihn befriedigt. Das bringt geistige Ruhe und } \\
\text { Ausgeglichenheit. }\end{array}$ \\
\hline D & Verlangen & $\begin{array}{l}\text { das Problem am besten zu lösen aber nicht um jeden } \\
\text { Preis. Das Verlangen ist das Ziel, das motiviert. }\end{array}$ \\
\hline AA & Aktivität & bedeutet eigene Fähigkeiten, Gewandtheiten, \\
Areativität und Phantasie zu nutzen.
\end{tabular}

Das Instrument für den Schulz der geistigen Gesundheit und guten Kondition der Manager ist so Teplická und Kameníková, die Wertpyramide der geistigen Gesundheit, die sie wie folgt darstellen:

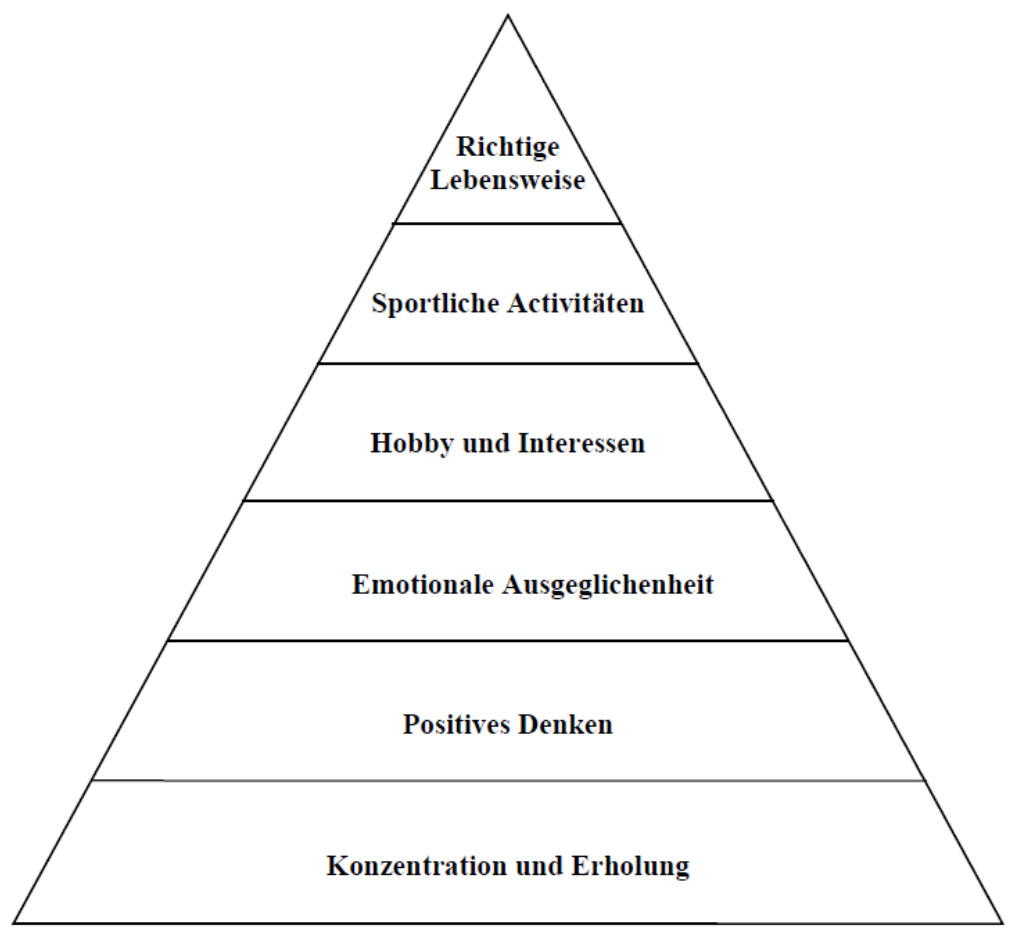




\subsection{Was kann ein Workaholic mit seiner Abhängigkeit tun?}

Arbeitssucht fordert ebenso wie jede andere Sucht seinen Tribut an der Gesundheit. Schlafdeprivation, Hypertension, Stress und erhöhtes Schlaganfall- oder Infarktrisiko gehen häufig Hand in Hand mit der Arbeitssucht (das Burnout-Syndrom als potentielle Folge der Arbeitssucht ist dann nicht selten von klinischen Depressionen begleitet).

Sich von Arbeitssucht zu befreien, ist wie die Befreiung von einer Drogensucht. Es handelt sich also keinesfalls um eine kurzfristige Angelegenheit. Wenn der Mensch die Kraft findet, sich behandeln zu lassen, ist vor allem die Unterstützung von der Umgebung wichtig. Aber auch nach einer langen Behandlung muss der Mensch noch nicht ganz gewonnen haben. Es genügt wenig und er kann dorthin gelangen, wo er bereits war. Wichtig ist deshalb, die ersten Anzeichen der Arbeitssucht zu erkennen.

Was die Heilung des Arbeitssucht anbelangt, so gibt es t eine breite Skala von Ansichten. Ramsey (A word to workaholics. Super Vision 2/2002) empfiehlt folgende Schritte:

- Erkennen sie Ihre Abhängigkeit. Machen Sie eine persönliche Inventur, bekennen sie anderen gegenüber ihre Fehler, machen sie sich eine Liste der Menschen, denen sie mit Ihrer Sucht Schaden zugefügt haben und bemühen sie sich um Besserung. Denken sie daran: das Wichtigste ist: arbeiten und leben.

- Setzen sie Grenzen. Das ist eine Art und Weise, sich bewusst zu machen, dass sie nicht alles allein machen können.

- Bemühen sie sich, jeden Tag rechtzeitig nach Hause zu kommen. Sie werden überrascht sein - die Welt geht nicht unter.

- Legen sie jeden Tag in der Arbeit mehrere Pausen ein.

- Ändern sie ihre Anschauungsweise über die Zeit. Zeit ist ein Instrument keinesfalls ein Feind.

- Vereinfachen sie sich Ihr Leben.

- Planen sie Ruhe ein und nehmen sie sich etwas Zeit nur für sich.

- Ändern sie Ihre Routine. Flexibilität ist das Gegengift der Arbeitssucht.

- Schauen sie so lange nicht auf die Uhr, wie sie es aushalten.

- Nehmen sie nicht an überflüssigen Meetings und Beratungen teil.

- Stellen sie sich reale Ziele.

- Nehmen sie eine beliebige Änderung vor, durch die sie ihre jetzige Auflage erleichtern. 
- Wenn sie der Vorstellung eines langen Urlaubs nicht standhalten können, versuchen sie es mit einem kurzen. Anfangs genügen auch ein paar Tage. Und wenn sie es aushalten, nicht zur Arbeit zu telefonieren, ist das das i-Tüpfelchen.

- Haben sie keine Angst, in der Arbeit um Hilfe zu bitten. Bemühen sie sich nicht, alles allein zu machen.

- Bemühen sie sich nicht, jede Arbeit vollkommen zu machen. Viele Aufgaben verdienen das einfach nicht.

- Arbeiten sie so schnell, wie sie können und so langsam, wie sie es für die Arbeit brauchen.

- Schreiben sie weniger Mitteilungen, sprechen sie lieber mit den Menschen.

- Schenken sie dem, was um sie herum geschieht, Aufmerksamkeit. Versuchen sie ,im Bilde" zu sein und das nicht nur ,in der Arbeit".

- Schauen sie nach neuen Gelegenheiten. Ziehen sie alle Möglichkeiten in Erwägung und versuchen sie etwas Anderes.

- Machen sie eine Liste ihrer eigenen Rollen (Ehemann/Ehefrau, Vater/Mutter, Sohn/Tochter, Sportfan, Kunstliebhaber u. ä.). Sie sind mehr als nur Ihre Arbeit.

- Widmen sie sich den Kindern.

- Denken sie daran, dass Sie für das Leben arbeiten und nicht umgekehrt.

- Kehren sie zu ihren Leidenschaften zurück, erinnern sie sich an vergessene Interessen. Kommt es ihnen merkwürdig vor, dass sie sie aufgegeben haben?

- Suchen sie sich irgendeinen ruhigen Ort und bleiben sie dort so lange, bis sie Ruhe gefunden haben.

- Nutzen sie Gruppentherapien.

- Lassen sie sich beraten oder suchen sie einen persönlichen Berater, der ihnen hilft, den Weg zu den Werten des Lebens zu finden.

- Nehmen sie die Dinge und sich selbst mit Humor. Das Leben ist zu ernst, als dass es auch noch ständig weiter nur ernst genommen wird.

- Wenn sie gläubig sind, versuchen sie Ihren Glauben zu beleben.

- Wenn sie denken, dass für ihre Arbeitssucht die Kultur Ihrer Firma verantwortlich ist, verlassen sie die Firma.

Oben aufgeführte Schritte sind keine Zauberformeln, aber können Wunder vollbringen und vielen Workaholics behilflich sein, die Arbeit und das Leben zu verbessern. 


\subsection{Was bedeutet das für die Praxis in der Firma}

Eine ganze Reihe von Unternehmen ist sich des schädlichen Einflusses der Arbeitssucht und ihrer Folgen bislang nicht voll bewusst. Die Vermehrung oder das Tolerieren von Arbeitssucht kompliziert nicht nur der Firma den Weg zur Erfüllung ihrer Unternehmensziele, sondern beeinflusst auf grundlegende Weise auch ihren Ruf als Arbeitgeber. Die Menschen können nämlich den Eindruck erhalten, dass übertriebene Arbeit in der Firma erwartet, geschätzt und bevorzugt wird. Das ist aber keine gute Visitenkarte für eine Firma, die neue Talente anlocken oder ihr Schlüsselpersonal halten will. Die Firma sollte also im Interesse strategischer Prosperität um die Schaffung eines Arbeitsumfeldes bemüht sein, das in höchstmöglichem Maße, das Entstehen und Tolerieren von Arbeitssucht ausschließt.

Was Führungskräfte und das Personal der Risikogruppen überhaupt anbelangt, hat die Praxis bewiesen, dass eine der häufigsten Ursachen des Arbeitssucht die Unfähigkeit dieser Personen ist, zwischen Dringlichkeit und Relevanz einer Sache zu unterscheiden und ihre Zeit dementsprechend auszurichten. Das führt zu einem falschen Umgang mit dem geistigen Kapital, und zwar dem eigenen, als auch dem der Mitarbeiter. Die zu den Risikogruppen zählenden Personen sollten deshalb im Rahmen ihrer Entwicklung keinesfalls das „time management vergessen oder unterschätzen. Eine häufige Ursache für die Arbeitssucht ist aber auch in der Erholung zu suchen. Müdigkeit und Erschöpfung sind typische Anzeichen für Überarbeitung. Besonders gefährlich ist geistige Müdigkeit chronischer Art, denn ein chronisch müder Mensch hat wenig Kraft (Energie), ist gereizt und schläfrig (oft deshalb, weil er nicht schlafen kann) und hegt quälende Gefühle, sofort wieder arbeiten zu müssen. Die einfachste Art, der Müdigkeit vorzubeugen und sie zu überwinden ist „R $\boldsymbol{u} \boldsymbol{h} \boldsymbol{e}$ Erholung". Müdigkeit durch Wille und Anspannung überwinden zu wollen, hat keinen Erfolg. Wirksam sind Rhythmusänderungen des Arbeitstages, eine ausgeglichene Organisation des Arbeitstages, die Einordnung von Pausen und der Wechsel von Tätigkeiten. Eine gute Lösung ist auch gleitende Arbeitszeit. Im Kontext mit der „Kunst, sich erholen zu können" gilt folgendes Prinzip: je mehr sich ein

Mensch mit Problemen befasst, um so mehr muss er sich erholen (es gibt eine ganze Skala von Erholungstechniken, wie z.B. autogenes Training, die Methode progressiver Erholung, Meditationstechniken etc., die dem Menschen helfen, unangenehme Spannungszustände zu überwinden und sich mit schwierigen Situationen auseinanderzusetzen zu können). 


\section{BURNOUT-SYNDROM - DER SCHRECKEN AMBIZIÖSER MENSCHEN}

Zweifelsohne ist die heutige Zeit viel komplizierter als je zuvor. Fusionen, Umgestaltungen und die damit verbundenen Entlassungen forcieren die Nervosität der Führungskräfte und übrigen Arbeitskräfte in den meisten Firmen. Die Menschen müssen oft eine Entscheidung in Arbeitsproblemen treffen oder Probleme klären, ohne jedoch über adäquate Kapital- und Informationsquellen oder Humanressourcen zu verfügen. Nach langwährenden Versuchen, nicht Machbares zu schaffen, sind einige von ihnen einfach ,ausgebrannt". Was heißt das aber?

Das Burnout-Syndrom kann als mentaler Zustand beschrieben werden, der häufig bei Menschen auftritt, die mit anderen Menschen arbeiten und deren Beruf von interpersoneller Kommunikation abhängig ist.

\subsection{Ursachen und Symptome des Burnout-Syndroms}

Menschen, die von ihrer Arbeit nicht mehr als Lohn erwarten, können Stress erleben aber gewöhnlich sind sie nicht vom Burnout-Syndrom betroffen (Stress ist nämlich nicht dasselbe wie das Burnout-Syndrom). Das Syndrom trifft in der Regel Individuen mit ausgezeichneten Qualitäten, die möchten, dass ihre Arbeit einen Sinn hat und die in ihr etwas beweisen wollen. Wenn sie feststellen, dass sie trotz aller Anstrengungen nichts ausrichten können, sind sie auf dem besten Wege zum „Ausbrennen".

Das Burnout-Syndrom ist die Folge lang anhaltender Stresszustände und einer unangebrachten Auseinandersetzung mit der psychischen und physischen Belastung. Unter chronischem Stress leiden insbesondere Menschen, die einer Dauerbelastung im sozialemotionellen Bereich ausgesetzt sind (eine starke Risikogruppe sind die Führungskräfte).

Die Ursache des Problems sind jedoch nicht nur anstrengende, überwiegend geistige Arbeit, viele Überstunden oder Entscheidungen und Arbeit unter Stress. Ursache sind auch nicht erfüllte Hoffnungen und eine nicht realisierte Aspiration hoch motivierter Menschen. Die Quintessenz des Burnout-Syndroms ist die Erschöpfung des menschlichen Willens.

Das Burnout-Syndrom ist eine schmerzhafte Erfahrung, die von physischen (körperlichen) und psychischen Symptomen begleitet ist und bei der auch die sozialen Beziehungen nicht abseits bleiben.

Typische physische Symptome sind: 
- Schlafstörungen, gesteigerter Appetit, Anfälligkeit für Krankheiten, vegetative Beschwerden (Herz, Atmung, Verdauung),

- Rasche Müdigkeit, Erschöpfung, Muskelspannungen, hoher Blutdruck. Häufigste psychische Symptome sind:

- Verlust an Enthusiasmus, Arbeitseinsatz, Verantwortung

- Appetitlosigkeit, Gleichgültigkeit gegenüber der Arbeit

- Negative Haltung gegenüber sich selbst, der Arbeit, Firma, Gesellschaft, dem Leben

- Entfliehen in die Phantasie

- Konzentrationsschwierigkeiten, Vergesslichkeit

- Niedergeschlagenheit, Hilflosigkeitsgefühle, Reizbarkeit, Aggressivität, Unzufriedenheit

- Gefühl ungenügender Anerkennung.

In den sozialen Beziehungen äußern sich die Symptome in der Regel in:

- abnehmendem Engagement, abnehmendem Bemühen, den Kunden zu helfen (insbesondere problematischen Kunden),

- der Einschränkung der Kontakte mit den Kollegen, Kunden,

- der Zunahme von Konflikten in der Arbeit und im Privatleben.

Der Prozess, der in einem Ausbrennen gipfelt, zieht sich manchmal über Monate, über Jahre hin. Er verläuft in mehreren Phasen, die unterschiedlich lang sein können. Auf die Praxis in der Firma bezogen, lässt sich der gesamte Prozess trefflich mit folgenden Phasen veranschaulichen:

- Begeisterung (hohe Ideale, starkes Engagement),

- Stagnation (die Umsetzung von Idealen misslingt, die Orientierung ändert sich, die Forderungen der Kunden oder des Arbeitgebers beginnen lästig zu werden),

- Frustration (dieser Mensch nimmt den Kunden negativ wahr, die Arbeitsstelle ist für ihn eine große Enttäuschung),

- Apathie (zwischen diesem Menschen und dem Kunden herrscht Feindschaft, dieser Mensch weicht Fachgesprächen mit den Kollegen und jeglichen Aktivitäten aus),

- Burnout-Syndrom (das Stadium der völligen Erschöpfung ist erreicht - es wird kein Sinn in der Arbeit gesehen, Zynismus, Depersonalisation, Entfremdung, Schwinden der Reflexe innerer Normen). 


\subsection{Wie kann man das Burnout-Syndrom beherrschen?}

Derjenige, der das Burnout-Syndrom erfolgreich besiegen will, sollte es zuerst mit einer Selbstanalyse versuchen. Denn die Bewertung der eigenen guten und schwachen Seiten kann die betroffene Person ins Leben zurückrufen. Das gilt insbesondere für diejenigen, die sich hohe Ziele setzen ( Manager ). Wichtig ist auch, sich darüber im Klaren zu sein, dass die Ursachen des Syndroms oft in einer schlechten Organisation der Arbeit liegen.

Empfehlungen und Anleitungen dafür, „was zu machen ist", um das Burnout-Syndrom in den Griff zu bekommen, gibt es viele. Stanley (Burnout: a managers's worst nightmare, 2001) rät:

- beschuldigen sie sich nicht selbst, das vertieft die Probleme nur noch mehr,

- schauen sie sich um und entscheiden sie, was sich ändern lässt und was nicht. Unternehmen sie dort, wo das möglich ist, die entsprechenden Schritte zur Ausschaltung der Stressfaktoren in der Arbeit,

- übernehmen sie keine weiteren Projekte und machen sie sich bewusst, dass die menschlichen Kräfte ihre Grenzen haben Versuchen sie nicht, ein Übermensch zu sein,

- versuchen sie soziale Beziehungen in der Arbeit und außerhalb von ihr einzugehen. Es ist wichtig, Freunde und Familienmitglieder zu haben, die helfen, die Probleme zu bewältigen,

- suchen sie sich Aktivitäten außerhalb der Arbeit. Dabei kann es sich um Erholung, Weiterbildung oder nur gewöhnliches Ausspannen handeln. Entscheidend ist, ob ihnen das hilft, Stress abzubauen,

- essen sie gesünder und machen sie regelmäßig Sport!

- wenn sie denken, dass sie das nicht alles allein schaffen können, suchen sie sich einen Berater aus Fachkreisen - einen Coach, der Ihnen hilft, eine langfristige Strategie für die Bewältigung des Burnout-Syndroms aufzustellen.

\subsection{Was bedeutet das für die Firmenpraxis?}

Die Art der Auseinandersetzung mit dem Burnout-Syndrom hängt in wesentlichem Maße von der konkreten Arbeit ab, die bei der betroffenen Person Ursache ihrer Beschwerden war (also mehr oder weniger ist sie individuell). Wenn es sich um Manager handelt (sie zählen zur risikoreichsten Gruppe), kommen in der Regel nachstehende drei Szenarien in Betracht:

- In der gleichen Firma auf eine weniger anspruchsvolle leitende (Manager) Funktion überwechseln. 
Das Risiko, dieser auf den ersten Blick logischen Alternative, besteht darin, dass sich die Beschwerden mit der Zeit auch auf diesem Posten äußern können, wenn die Ursachen, die das Syndrom ausgelöst haben, nicht behoben werden.

- Auf eine höhere leitende (Manager) Funktion überwechseln und somit dem Chaos und den Problemen, die untere und mittlere Führungsebenen beschäftigen, aus dem Wege gehen.

Diese Lösung macht die Situation nur noch komplizierter und schlechter, da die Führungskraft, die am Burnout-Syndrom leidet, auf einer höheren Stufe nicht in der Lage sein wird, schwierige Entscheidungen zu treffen.

\section{- Die leitende (Manager) Funktion niederlegen und die Firma verlassen.}

Eine äußerst schmerzende Lösungsvariante und dies sowohl für den Manager, als auch für die Firma. Manager sind es in der Regel nicht gewohnt, aufzugeben und eine Niederlage zuzugeben bzw. zu erleben und die Firma verliert mit der abtretenden Führungskraft auch deren Fähigkeiten und Kenntnisse.

Der aktuelle Appell an die Führungskräfte (Manager) lautet nach Stanley (Burnout: a manager's worst nightmare, 2002): lernen, den Stress in einen motivierenden Faktor für die persönliche und berufliche Weiterentwicklung $\mathrm{zu}$ transformieren. Dadurch können die Führungskräfte (Manager) erfolgreicher werden und für die Firma wird dies von erheblichem Nutzen sein. Das setzt aber voraus, dass die leistungsstärksten Manager von den Firmen unterstützt werden (hoch motivierte Manager sollten angemessene Unterstützung bei der Umsetzung ihrer Ziele erhalten).

\section{FAZIT}

Arbeitssucht ist schädlich. Krankhaft arbeitsame Manager fordern gleiches Verhalten nicht selten auch von den ihnen unterstellten Mitarbeitern, was bei ihnen früher oder später zu Zorn und Demoralisierung führt. Am Arbeitssucht leidet natürlich nicht nur der Betroffene selbst. Ein ewig beschäftigter Manager ist meist kein guter Manager und in der Regel ist er auch nicht für das Familienleben geschaffen. Viele Workaholics leiden deshalb auch unter dem Zerfall der Familie. Workaholics verlieren jedoch nicht nur die Familie, sondern auch das eigene Leben - sie existieren und arbeiten aber nur wenige von ihnen leben. Heilung ist möglich, in der Regel aber schwierig und nur selten kommt sie ohne fachliche Hilfe aus. Der Betroffene will nämlich oft über lange Zeit hinaus nicht zugeben, dass er süchtig ist und lehnt alle rationellen Argumente seiner Umgebung mit aller Schärfe ab. Der beste Schutz ist Vorbeugung, d.h. das Leben mannigfaltig zu gestalten und sich zu 
fragen, weshalb das oder jenes eigentlich gemacht werden muss, welchen Sinn hat das und worin besteht das Ziel.

Das Burnout-Syndrom ist in der Regel ein schleichender Prozess und seiner Entstehung geht hoher Arbeitseinsatz voraus, durch den der Betroffene die besten Resultate erzielen will (deshalb sind die Betroffenen meist hoch motivierte und ihrer Arbeit völlig ergebene Menschen). Die negative und zynische Haltung, die gewöhnlich eine Begleiterscheinung ist, hat dann oft ernsthafte Auswirkungen auf die Arbeit, Kundendienste, Kollegen, Unterstellte und Vorgesetzte. Das Burnout-Syndrom kann sich aber auch in einer schlechten Arbeitsmoral, Unpünktlichkeit, häufigem Wechsel des Arbeitgebers oder der Arbeit selbst äußern. Die betroffene Person gerät leicht in Konflikte, verliert Freunde und hat auch im familiären Leben Probleme. Man kann das Burnout-Syndrom beherrschen aber manchmal ist ein Arbeitswechsel, um Stresssituationen aus dem Weg zu gehen, unumgänglich. Das beste Heilmittel ist jedoch Prävention. Deshalb muss die Firma ihren risikogefährdeten Mitarbeiter im Kampf gegen überflüssigen Stress und das Burnout-Syndrom unterstützen. Es zahlt sich ihnen aus, wenn sie die Schwierigkeiten dieser Mitarbeiter anerkennen, ihnen günstige Arbeitsbedingungen schaffen und sie redlich nach ihren Leistungen entlohnen.

\section{Literatur}

ARMSTRONG, M. Personální management. (Personalmanagement) Grada, Praha, 1999 CIBIS, V. a kol. Člověk. (Der Mensch) Scientia, Praha, 1996

ČIŽKOVÁ, J. a kol. Poznávání duševního života člověka. (Kennenlernen des menschlichen geistigen Lebens.) Univerzita Palackeho (Palacky-Universität), Olomouc, 1997

DRÄPELA, V.J. Přehled teorií osobnosti. (Übersicht der Persönlichkeitstheorien.) Portal, Praha, 1998

PROŠKO, J. a kol. Poruchy osobnosti. (Persönlichkeitsstörungen.) Portal, Praha, 2003

RUSH, M. D. Syndrom vyhoření. (Burnout-Syndrom.) Návrat domü, Praha, 2003

RAMSEY, R.R. A word to workaholics. Super Vision 2/2002

STANLEY, T.L. Burnout: a manager's worst nightmare. Super Vision 8/2001

STANLEY, T.L. Syndrom vyhořeni. (Burnout-Syndrom.) Moderni řizeni č.8 (Modernes Management Nr. 8), Praha, 2005

TEPLICKÁ, K., KAMENÍKOVÁ, K. Duševni zdravi predevším. (Geistige Gesundheit - das A und O) Moderni řizeni č.7 (Modernes Management Nr. 7), Praha, 2004 